Please share your stories about how Open Access to this article benefits you.

\title{
Demonstration of Geometric Landau-Zener Interferometry in a Superconducting Qubit
}

by Xinsheng Tan et al.

2013

This is the published version of the article, made available with the permission of the publisher. The original published version can be found at the link below.

Xinsheng Tan et al. (2014). Demonstration of Geometric Landau-Zener Interferometry in a Superconducting Qubit. Phys. Rev. Lett. 112:27001.

Published version: http://dx.doi.org/10.1103/PhysRevLett.112.027001

Terms of Use: http://www2.ku.edu/ scholar/docs/license.shtml 


\title{
Demonstration of Geometric Landau-Zener Interferometry in a Superconducting Qubit
}

\author{
Xinsheng Tan, ${ }^{1,2}$ Dan-Wei Zhang, ${ }^{3}$ Zhentao Zhang, ${ }^{1, *}$ Yang Yu, ${ }^{1, \dagger}$ Siyuan Han, ${ }^{2, \$}$ and Shi-Liang Zhu ${ }^{1,3, \S}$ \\ ${ }^{1}$ National Laboratory of Solid State Microstructures, School of Physics, Nanjing University, Nanjing 210093, China \\ ${ }^{2}$ Department of Physics and Astronomy, University of Kansas, Lawrence, Kansas 66045, USA \\ ${ }^{3}$ Laboratory of Quantum Engineering and Quantum Materials, SPTE, South China Normal University, Guangzhou 510006, China \\ (Received 4 April 2013; revised manuscript received 10 July 2013; published 14 January 2014)
}

\begin{abstract}
Geometric quantum manipulation and Landau-Zener interferometry have been separately explored in many quantum systems. In this Letter, we combine these two approaches to study the dynamics of a superconducting phase qubit. We experimentally demonstrate Landau-Zener interferometry based on the pure geometric phases in this solid-state qubit. We observe the interference caused by a pure geometric phase accumulated in the evolution between two consecutive Landau-Zener transitions, while the dynamical phase is canceled out by a spin-echo pulse. The full controllability of the qubit state as a function of the intrinsically robust geometric phase provides a promising approach for quantum state manipulation.
\end{abstract}

Solid-state devices based on superconducting Josephson junctions present an excellent platform for exploring quantum mechanics and hold promise for applications in quantum information processing [1]. Great successes have been achieved in demonstrating the quantum coherent nature of these artificial atoms [2-18]. However, in order to realize practical quantum computation, much research is still needed to find the optimal balance between controllability and decoherence.

A promising approach to achieve robust control of a quantum system is by using geometric phases which arise from a cyclic evolution of the quantum system [19-27]. These geometric phases have been observed in a wide variety of systems including superconducting qubits [27-29]. In particular, the robustness of geometric phases against certain noises [30,31] has been confirmed in a recent experiment based on a superconducting qubit [32]. One of the most promising applications of geometric phases on quantum control is geometric quantum computation. The design of fault-tolerant quantum logic gates is the central issue in realizing quantum computation. The phases in a quantum gate are usually a combination of geometric phases and dynamical phases. The performances of these two types of phases are essentially different: in contrast to dynamical phases which are sensitive to the rate of passage, geometric phases depend solely on the global geometry of the trajectory traversed by the state of the system. Because geometric phases are immune to certain local fluctuations, it was proposed that quantum gates with purely geometric phases, which are called geometric quantum gates [20,21], are intrinsically fault tolerant [22-25]. Interestingly, high-fidelity geometric quantum gates have been experimentally realized by several groups $[26,27]$.

On the other hand, the Landau-Zener (LZ) transition (LZT) has been demonstrated to be another important approach to control a quantum system [33-38]. When a quantum two-level system is driven through an avoided energy-level crossing, the system can undergo nonadiabatic transitions with the LZT probability

$$
P_{\mathrm{LZ}}=\exp \left(-\pi \Delta^{2} / 2 \hbar v\right),
$$

where $\Delta$ is the energy gap at the avoided crossing and $v$ is the speed of energy variation [33]. Quantum interference and manipulations of qubit states based on this phenomenon have been reported in different quantum systems [33-38]. However, in these studies, the dynamical phases dominate the entire evolution. In other words, while LZ interferometry and geometric quantum manipulation are both well explored in many quantum systems, they have not been jointly examined in detail. In a recent interesting Letter [39], the manipulation of geometric phases with LZT in a superconducting charge qubit was theoretically proposed; however, no experimental observation has been reported so far.

In this Letter, we fill this gap by reporting an experiment that combines the LZT and geometric phase manipulation. We design and experimentally realize LZ interferometry with pure geometric phases in a superconducting phase qubit. Since the dynamics of a superconducting qubit coupled to microwave fields is analogous to the dynamics of a spin- $1 / 2$ particle subjected to a rotating magnetic field, all kinds of geometric phases may accumulate in a designed evolution [19]. We observe the oscillations of the excited state population versus the geometric phase, which is an ubiquitous signature of quantum interferences. Our numerical simulation results using the measured energy relaxation and phase decoherence times agree well with the experimental data, confirming the observation of the geometric LZ interference. Furthermore, the observed contrast of LZ interferences with pure geometric phases is higher than that of dynamical LZ interferences, which shows the robustness of the geometric LZ interferometry (GLZI). Since LZ interferences have been proposed to have many 
applications in various quantum systems [33], the demonstrated GLZI in our experiment paves a new way to achieve reliable control of qubit states. Therefore, our work should shed light on and stimulate interests in applying LZ interferometry for quantum state control in general and faulttolerant quantum information processing in particular.

The system used to demonstrate GLZI is a flux-biased superconducting phase qubit. The truncated Hamiltonian of the lowest two levels $(|0\rangle,|1\rangle)$ in the energy bases is $H_{q}=\frac{1}{2} \hbar \omega \sigma_{z}[38,40]$, where $\sigma_{z}$ is the Pauli operator in the $z$ direction (see the Supplemental Material [41]). $\hbar \omega$ denotes the energy difference between $|0\rangle$ and $|1\rangle$, which could be tuned by varying the external magnetic flux $\phi_{e x}$ threading the loop, as illustrated in Fig. 1(a). Moreover, the state of the qubit can be controlled by microwave (MW) irradiation. With a MW field, the Hamiltonian becomes $H^{\prime}=\frac{1}{2} \hbar \omega \sigma_{z}+\hbar \Omega \cos \left(\omega_{m} t+\theta\right) \sigma_{x}$, where $\Omega$ is the Rabi frequency proportional to the amplitude of the MW, $\omega_{m}$ $(\theta)$ is the frequency (phase) of the MW. With respect to the frame rotating with frequency $\omega_{m}$, we apply the rotating wave approximation to obtain

$$
H_{\mathcal{B}}=\frac{1}{2} \hbar \delta \sigma_{z}+\frac{1}{2} \hbar \Omega_{x} \sigma_{x}+\frac{1}{2} \hbar \Omega_{y} \sigma_{y} \equiv \hbar \vec{B} \cdot \frac{\vec{\sigma}}{2},
$$

where $\delta=\omega-\omega_{m}$ is the detuning, $\Omega_{x}=\Omega \cos \theta$, and $\Omega_{y}=\Omega \sin \theta$. The dynamics of the qubit is thus identical (a)
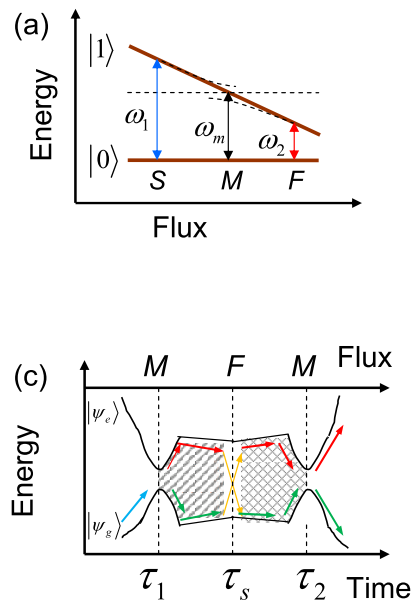
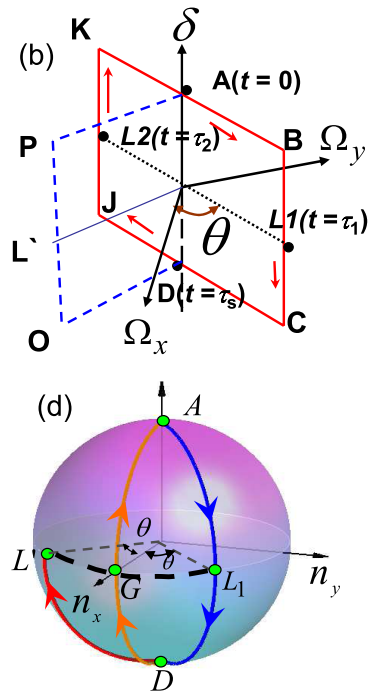

FIG. 1 (color online). (a) The qubit eigenenergies (solid lines) $E_{0,1}$ (with respect to ground state energy $E_{0}$ ) versus flux bias. An effective avoided crossing (dashed lines) related to $H_{\mathcal{B}}$ is created at point $M$ by the MW with frequency $\omega_{m}$. (b) Effective magnetic fields. The trajectory along $A B C D J K A(A B C D O P A)$ is considered in this Letter (Ref. [39]). (c) The corresponding instantaneous eigenvalues of $H_{\mathcal{B}}$ versus time and flux bias. The qubit is initially in the ground state $\left|\psi_{g}\right\rangle=|0\rangle$, which splits at time $t=\tau_{1}$ during the first LZT, evolving along two different paths and interfering at time $t=\tau_{2}$ of the second LZT. At point $F$, the state is flipped by a $\pi$ pulse to eliminate the dynamical phase. (d) The Bloch vectors of the qubit in the GLZI considered in Ref. [39] and here. to that of a spin- $1 / 2$ particle subjected to an effective magnetic field $\vec{B}=\left(\Omega_{x}, \Omega_{y}, \delta\right)$. Therefore, the geometric phases may accumulate in a designed evolution [19].

The Hamiltonian $H_{\mathcal{B}}$ is essentially identical to that in Ref. [39] and can therefore be used to realize GLZI. In the following, we briefly summarize GLZI proposed in Ref. [39] and our modifications for implementation in a superconducting phase qubit. In the original proposal in Ref. [39], the dynamical phase was not removed. Our method improved upon this by eliminating both the dynamical phase and the Stokes phase based on a spin-echo method, which was used to remove dynamical phases in geometric quantum computation [21,23]. To illustrate our scheme, we plot the effective magnetic fields in Fig. 1(b), where the trajectory along $A B C D O P A$ is considered in Ref. [39] and the path along $A B C D J K A$ is studied here. In Fig. 1(c), we plot the energies of the adiabatic states $|g\rangle,|e\rangle$ as a function of time in a GLZI, obtained by instantaneous diagonalization of $H_{\mathcal{B}}$. The LZTs occur at the avoided level crossings at time $t=\tau_{1}, \tau_{2}$. The probability of a nonadiabatic LZT at such a crossing is given approximately by Eq. (1), where $\Delta=\Omega$ and $v=\hbar\left(\omega_{1}-\omega_{2}\right) / \tau_{p}$, with $\tau_{p}$ being the LZ sweeping time defined here as the time swept from point $S$ to point $F$ in Fig. 1(a). Here, $\omega_{1}\left(\omega_{2}\right)$ is the energy level spacing at point $S(F)$. Comparing our Figs. 1(b),(c) with Figs. 1 (b),(c) in Ref. [39], we make two modifications. One is that the trajectory of effective field is now a rectangle. The other modification, which is a substantial improvement, is the use of a spin echo at time $\tau_{s}$ to remove the dynamical phase [42] as well as to make the evolution between two LZs a closed path. To illustrate the latter point, we plot the evolution path $n_{j}^{g}=\left\langle\psi_{g}\left|\sigma_{j}\right| \psi_{g}\right\rangle \quad(j=x, y, z)$ with initial ground state $\mid \psi_{g}\left(t=\tau_{1}\right)$ between two LZTs on the Bloch sphere in Fig. 1(d) under the condition of adiabatic approximation. The path $L_{1} D L^{\prime}$ is for the trajectory studied in Ref. [39], where the geometric phase is $\theta$ determined by the spherical triangle $L_{1} D L^{\prime}$. On the other hand, the trajectory of $n_{j}^{g}$ considered here is along the closed path $L_{1} D G A L_{1}$ in Fig. 1(d). The trajectory $L_{1} D$ of the evolution follows the magnetic field $L_{1} C D$ in Fig. 1(b). The spin echo leads the Bloch vector to evolve alone $D G A$, and finally the Bloch vector evolves along $A L_{1}$ (as an excited state) when the magnetic field moves along $D J L_{2}$ in Fig. 1(b). Therefore, the geometric phase accumulated between the two LZ transitions is also $\theta$ determined by the trajectory $L_{1} D G A L_{1}$ in Fig. 1(d).

Following the adiabatic-impulse method outlined in Refs. $[33,34,39]$, we obtain the population on the excited state after the entire evolution,

$$
P_{1}=1-4 P_{\mathrm{LZ}}\left(1-P_{\mathrm{LZ}}\right) \sin ^{2} \theta .
$$

It is clear from Eq. (3) that $P_{1}$ is independent of the dynamical phase and hence insensitive to fluctuations in qubit 
transition frequency $\omega(t)=E_{1}(t)-E_{0}(t)$. Furthermore, comparing with that in Ref. [39], we find that the intereference pattern resulting from our method is independent of the Stokes phase [43]. Therefore, our GLZI scheme should be more robust against various harmful dynamical effects.

We use a trilayer $\mathrm{Al} / \mathrm{AlO}_{x} / \mathrm{Al}$ superconducting phase qubit fabricated on crystalline $\mathrm{Al}_{2} \mathrm{O}_{3}$ substrate to implement our scheme experimentally. Figure 2(a) shows the principle circuitry of the qubit and its measurement. The qubit is cooled to $30 \mathrm{mK}$ in a dilution refrigerator. We measure the energy relaxation time $T_{1}$ and decoherence time $T_{2}$ of the qubit using the standard pump probe and spin echo, respectively [40], and the results are $T_{1}=118 \pm 3$ and $T_{2}=157 \pm 11 \mathrm{~ns}$ [41].

Figure 2(b) shows the temporal profile of the flux bias and MW fields utilized to realize GLZI. In our experiment, the energy level spacing at points $S$ and $F$ are 14.4 and 14.2 GHz, respectively [Fig. 1(a)]. The frequency of MW1, which creates the avoided crossing, is $14.3 \mathrm{GHz}$. The size of the energy gap $\Omega$ at the avoided crossing can be tuned by adjusting the amplitude of MW1 [44]. We control the Hamiltonian $H_{\mathcal{B}}$ along a closed loop in the field $\vec{B}$ [Fig. 2(b)]. The $z$ component of the field is controlled by the flux bias, and $\Omega_{x}$ and $\Omega_{y}$ are controlled using an IQ mixer by adjusting the voltage level applied to the $I$ and $Q$ channels of the mixer. At $t=\tau_{s}$, we use MW2 to apply a $\pi$ pulse to the qubit, which causes the qubit to evolve along the path $D G A$ in Fig. 1(d). The pulse width is only several nanoseconds, which is much smaller than $\tau_{p}$, so it is reasonable to approximate it by an instant $\pi$ pulse in our numerical simulation. The $\theta$ angle in Fig. 1(b) and Fig. 1(d) is determined by the ratio between the in-phase and quadrature components of the microwave. Finally, after the second LZT is completed, a short readout pulse is applied to make a projective measurement of the qubit state.

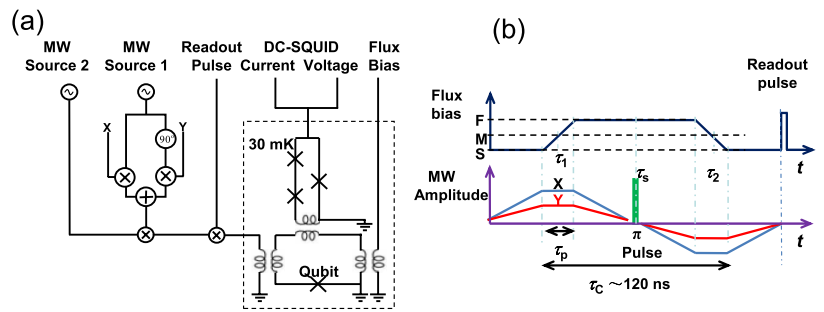

FIG. 2 (color online). (a) Schematic circuit of the phase qubit and its experimental setup. The crosses in the dashed box represent Josephson junctions. Magnetic flux bias line, microwave, readout control lines, and dc superconducting quantum interference device (SQUID) are inductively coupled to the qubit. An inphase and quadrature (IQ) mixer is used to control the $X$ and $Y$ components of MW1. (b) Schematics of the temporal profile of waveforms used to perform GLZI. An avoided crossing is created at point $M$ by MW1 [lower panel: blue or dark gray (red or light gray) line for the $X(Y)$ component]. MW2 produces the $\pi$ pulse at $t=\tau_{s}$ to null out the dynamical phase. The symbols $S, M$, and $F$ correspond to those in Fig. 1(a).
Figure 3 shows population $P_{1}$ as a function of the geometric phase and the LZ sweeping time $\tau_{p}$. As expected from Eq. (3), population $P_{1}$ is observed to oscillate sinusoidally as a function of the geometric phase. However, as shown in Fig. 3(a), the amplitude of the observed oscillation is substantially smaller than that predicted by Eq. (3). We noticed that some approximations, such as the adiabatic-impulse approximation and the asymptotic value of $P_{\text {LZ }}$ given by Eq. (1), are applied to obtain Eq. (3), whereas the decoherence and energy relaxation are not considered. To confirm that the observed oscillation is indeed caused by the geometric phase of the system, in the following we first discuss in which regime our experiment is performed. Since $H_{\mathcal{B}}$ is cyclic with a period around $\tau_{C} \sim 100 \mathrm{~ns}$, which is defined as the time spent traversing the cyclic path $S M F M S$, we may consider that the qubit is actually driven by an ac field with frequency $\omega_{C} / 2 \pi=1 / \tau_{C} \sim 10 \mathrm{MHz}$. Thus our experiment is in the region of $\Delta / \omega_{C} \sim 2$, whereas the previous experiments that investigated LZTs are in the limits of $\omega_{C} \gg \Delta$ [35] or $\omega_{C} \ll \Delta$ [36]. In addition, to verify the validity of those approximations used in Eq. (3), we numerically solve the Schrödinger equation with the Hamiltonian (2), and the result is shown as the red dotted line in Fig. 3(a). Comparing this line with the black dashed line obtained from the analytical expression given by Eq. (3) with $P_{\mathrm{LZ}} \simeq 0.61$ [which is
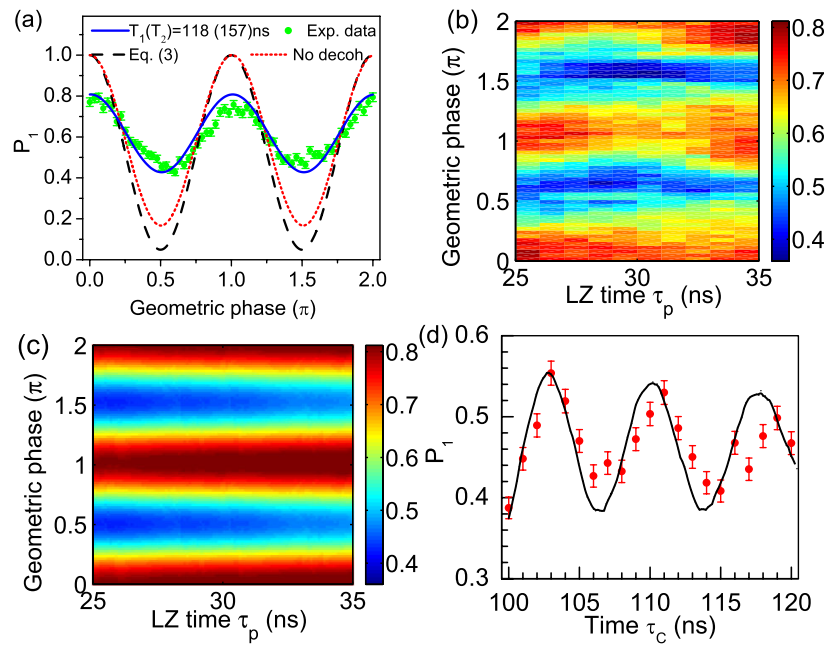

FIG. 3 (color online). (a) Population $P_{1}$ versus geometric phase with $\tau_{p}=25 \mathrm{~ns}$ and $\tau_{C}=100 \mathrm{~ns}$. Green dots are experimental data, while the black dashed line, the red dotted line, and the blue solid line are theoretical results obtained from Eq. (3), by numerically solving the Schrödinger equation and the master equation (4) with decoherence $T_{1}=118 \mathrm{~ns}$ and $T_{2}=157 \mathrm{~ns}$, respectively. The color scale represents (b) measured $P_{1}$, and (c) simulated $P_{1}$ with the master equation versus geometric phase and LZ period time $\tau_{p}$ for $\tau_{C}=100 \mathrm{~ns}$. (d) Measured population $P_{1}$ versus time $\tau_{C}$ for $\tau_{p}=25 \mathrm{~ns}$ in dynamical LZ interferometry. The solid line is the numerical simulation of the master equation. Other parameters: $\Delta / 2 \pi=\Omega / 2 \pi=$ $20 \mathrm{MHz}$ and $\delta / 2 \pi=100 \mathrm{MHz}$. 
derived by Eq. (1) for the parameters $\Delta / 2 \pi=20 \mathrm{MHz}$, $\left(\omega_{1}-\omega_{2}\right) / 2 \pi=200 \mathrm{MHz}$ and $\tau_{p}=25 \mathrm{~ns}$, the difference around $\theta=n \pi$ with $n$ an integer is negligible. On the other hand, around $\theta=(n+1 / 2) \pi$ the difference between the numerical and analytical results is relatively larger. To understand and resolve this apparent discrepancy, we plot the time evolution of population $P_{1}$ in Fig. S4 in the Supplemental Material [41] by numerically solving the Schrödinger equation for the cases of $\theta=0$ and $\theta=\pi / 2$, respectively. Population $P_{1}$ saturates after the first LZT, which is a clear-cut signature that the adiabaticimpulse model is valid under the experimental conditions. However, the saturation value $P_{\mathrm{LZ}}^{\prime} \simeq 0.71$ is not equal to $P_{\mathrm{LZ}} \simeq 0.61$ as directly derived from Eq. (1). After we use $P_{\mathrm{LZ}}^{\prime}$ to replace $P_{\mathrm{LZ}}$ in Eq. (3), we obtain a line which is almost identical to the red dotted line in Fig. 3(a). Therefore, we confirm that for our experiment, the adiabatic-impulse model is valid; however, the $P_{\mathrm{LZ}}$ in Eq. (1) should be modified under the experimental conditions.

Now the question is why the oscillation amplitude of the experimental result is significantly smaller than that of the numerical (analytical) results while they have the same oscillatory dependence of $P_{1}$ on the geometric phase $\theta$. As demonstrated below, the quantitative discrepancy between the experiment and calculations is due to energy relaxation and decoherence. Because $T_{1}$ and $T_{2}$ of the qubit are comparable to $\tau_{C}$, the effect of qubit decoherence cannot be ignored. In order to have a meaningful comparison between experiment and theory, we take the effects of finite $T_{1}$ and $T_{2}$ into consideration by numerically solving the master equation. The quantum dynamics of the system is thus described by the master equation of the time evolution of the density matrix $\rho$,

$$
\dot{\rho}=-\frac{i}{\hbar}\left[H_{\mathcal{B}}, \rho\right]-\Gamma[\rho],
$$

where the system Hamiltonian $H_{\mathcal{B}}$ is given by Eq. (2), and the second term, $\Gamma[\rho]$, describes the relaxation and dephasing processes phenomenologically. In a concrete expression, Eq. (4) can be rewritten as $\dot{\rho}_{11}=$ $-i\left[(\Omega / 2)\left(e^{-i \theta} \rho_{01}-e^{i \theta} \rho_{10}\right)\right]-\Gamma_{1} \rho_{11}, \dot{\rho}_{10}=-i\left[(\delta / 2) \rho_{10}+\right.$ $\left.(\Omega / 2) e^{-i \theta}\left(\rho_{00}-\rho_{11}\right)\right]-\gamma \rho_{10}, \dot{\rho}_{00}=-\dot{\rho}_{11}$ with $\rho_{01}=\rho_{10}^{\star}$, $\Gamma_{1} \equiv 1 / T_{1}$, and $\gamma \equiv 1 / T_{2}$.

The result obtained by solving the master equation without decoherence is identical to that obtained by numerically solving the Schrödinger equation. Furthermore, the simulated result using the measured qubit $T_{1}=118 \mathrm{~ns}$ and $T_{2}=157 \mathrm{~ns}$, shown as the blue solid line in Fig. 3(a), agrees fairly well with the experimental data. In addition, from Eq. (1), we know that by adjusting the sweeping speed of the flux bias $v=\hbar\left(\omega_{1}-\omega_{2}\right) / \tau_{p}$, one could change the transition probability $P_{\mathrm{LZ}}$. However, since the geometric phase does not depend on $P_{\mathrm{LZ}}$, the period and phase of $P_{1}(\theta)$ oscillation should be insensitive to changes in $v$
[Fig. 3(c)]. In Fig. 3(b), we show the measured $P_{1}(\theta)$ oscillation for the different LZ sweeping time $\tau_{p}$, which agrees quite well with the numerical results. Moreover, unlike the LZ interference of accumulated dynamical phases, $P_{1}$ does not oscillate with $\tau_{p}$, indicating that the dynamical phase is well eliminated by the spin-echo pulse.

Finally, we compare the performance of the geometric LZ interference with that of the conventional dynamical LZ interference. The measured $P_{1}$ as a function of the cyclic time $\tau_{C}$ for $\tau_{p}=25 \mathrm{~ns}$ is shown in Fig. 3(d). For dynamical LZ interferometry, the temporal profiles of the flux bias and MW fields are the same as those depicted in Fig. 2(c), except that no $\pi$ pulse is applied at point $F$. The phase angle $\theta$ of the microwave is kept constant, while $\tau_{C}$ is varied by changing the time spent in the plateau part of Fig. 2(c). Contrary to that of the geometric LZ interference, $P_{1}$ oscillates notably when $\tau_{C}$ is varied. More importantly, for the similar range of time $\tau_{C} \sim 100 \mathrm{~ns}$, the contrast (defined as the difference between the maximum and the minimum amplitudes of the oscillation) of the dynamical $\mathrm{LZ}$ interference is about 0.16 , which is much weaker than the corresponding value of 0.4 for the geometric LZ interference. Because $4 P_{\mathrm{LZ}}\left(1-P_{\mathrm{LZ}}\right)[33]$ is also the theoretical prediction for the contrast of dynamical LZ interferences, this result demonstrates that the geometric phase is more robust and stable against a variety of error-causing dynamical processes such as those caused by microscopic twolevel systems coupled to the qubit $[38,45]$.

In summary, we have experimentally demonstrated GLZI in a superconducting qubit. Our results indicate that GLZI may have advantages in many applications involving quantum state manipulation [33] such as simulating the timereversal symmetry with LZ interferometry [46]. Since our geometric LZ approach combines the simplicity of LZ interferometry and the robustness of geometric phases for quantum state manipulation, it may very well open a new venue for fault-tolerant quantum information processing.

We thank X. Wen, G. Sun, B. Mao, and M. Gong for their helpful discussion. This work is supported by the SKPBR of China (Grants No. 2011CB922104 and No. 2011CBA00200), NSFC (Grants No. 91021003, No. 11274156, and No. 11125417), NSF of Jiangsu (BK2010012), PAPD, and the PCSIRT. S. Han is supported in part by the U.S. NSF (PHY-1314861). We gratefully acknowledge Northrop Grumman ES in Baltimore, MD, for foundry support and thank R. Lewis, A. Pesetski, E. Folk, and J. Talvacchio for technical assistance. X. T., D.-W.Z., and Z.Z. contributed equally to this work.

\footnotetext{
*Present address: School of Physics Science and Information Engineering, Liaocheng University, Liaocheng 252059, China.

yuyang@nju.edu.cn

†Han@ku.edu
} 
§slzhu@scnu.edu.cn

[1] Y. Makhlin, G. Schön, and A. Shnirman, Rev. Mod. Phys. 73, 357 (2001).

[2] Y. Nakamura, Y. A. Pushkin, and J.S. Tsai, Nature (London) 398, 786 (1999).

[3] D. Vion, A. Aassime, A. Cottet, P. Joyez, H. Pothier, C. Urbina, D. Esteve, and M. H. Devoret, Science 296, 886 (2002).

[4] Y. Yu, S. Han, X. Chu, S. -I. Chu, and Z. Wang, Science 296, 889 (2002).

[5] I. Chiorescu, Y. Nakamura, C. J. P. M. Harmans, and J. E. Mooij, Science 299, 1869 (2003).

[6] J. Claudon, F. Balestro, F. W. J. Hekking, and O. Buisson, Phys. Rev. Lett. 93, 187003 (2004).

[7] R. McDermott, R. W. Simmonds, M. Steffen, K. B. Cooper, K. Cicak, K. D. Osborn, S. Oh, D. P. Pappas, and J. M. Martinis, Science 307, 1299 (2005).

[8] A. J. Berkley, H. Xu, R. C. Ramos, M. A. Gubrud, F. W. Strauch, P. R. Johnson, J. R. Anderson, A. J. Dragt, C. J. Lobb, and F. C. Wellstood, Science 300, 1548 (2003).

[9] M. Steffen, M. Ansmann, R. C. Bialczak, N. Katz, E. Lucero, R. McDermott, M. Neeley, E. M. Weig, A. N. Cleland, and J. M. Martinis, Science 313, 1423 (2006).

[10] J. H. Plantenberg, P. C. de Groot, C. J. P. M. Harmans, and J. E. Mooij, Nature (London) 447, 836 (2007).

[11] A. Wallraff, D. I. Schuster, A. Blais, L. Frunzio, R.-S. Huang, J. Majer, S. Kumar, S. M. Girvin, and R. J. Schoelkopf,, Nature (London) 431, 162 (2004).

[12] I. Chiorescu, P. Bertet, K. Semba, Y. Nakamura, C. J. P. M. Harmans, and J.E. Mooij., Nature (London) 431, 159 (2004).

[13] J. Mayer et al., Nature (London) 449, 443 (2007).

[14] M. A. Sillanpaa, J. I. Park, and R. W. Simmonds, Nature (London) 449, 438 (2007).

[15] C. M. Wilson, G. Johansson, A. Pourkabirian, M. Simoen, J. R. Johansson, T. Duty, F. Nori, and P. Delsing, Nature (London) 479, 376 (2011).

[16] H. Paik et al., Phys. Rev. Lett. 107, 240501 (2011).

[17] C. Rigetti et al., Phys. Rev. B 86, 100506 (2012).

[18] R. Vijay, C. Macklin, D. H. Slichter, S. J. Weber, K. W. Murch, R. Naik, A. N. Korotkov, and I. Siddiqi, Nature (London) 490, 77 (2012).

[19] M. V. BerryProc. R. Soc. A 392, 45 (1984).

[20] P. Zanardi and M. Rasetti, Phys. Lett. A 264, 94 (1999); S. L. Zhu and Z. D. Wang, Phys. Rev. Lett. 91, 187902 (2003).

[21] A. Ekert, M. Ericsson, P. Hayden, H. Inamori, J. A. Jones, D. K. L. Oi, and V. Vedral, J. Mod. Opt. 47, 2501 (2000).

[22] For a review on geometric quantum computation, see E. Sjöqvist, Physics 1, 35 (2008).

[23] J. A. Jones, V. Vedral, A. Ekert, and G. Castagnoli, Nature (London) 403, 869 (2000).

[24] L. M. Duan, J. I. Cirac, and P. Zoller, Science 292, 1695 (2001).
[25] G. Falci, R. Fazio, G. Palma, J. Siewert, and V. Vedral, Nature (London) 407, 355 (2000).

[26] D. Leibfried et al., Nature (London) 422, 412 (2003); J. Du, P. Zou, and Z. D. Wang, Phys. Rev. A 74, 020302(R) (2006).

[27] A. A. Abdumalikov, J. M. Fink, K. Juliusson, M. Pechal, S. Berger, A. Wallraff, and S. Filipp, Nature (London) 496, 482 (2013); G. Feng, G. Xu, and G. Long, Phys. Rev. Lett. 110, 190501 (2013).

[28] P. J. Leek, J. M. Fink, A. Blais, R. Bianchetti, M. Goppl, J. M. Gambetta, D. I. Schuster, L. Frunzio, R. J. Schoelkopf, and A. Wallraff, Science 318, 1889 (2007).

[29] M. Möttönen, J. J. Vartiainen, and J. P. Pekola, Phys. Rev. Lett. 100, 177201 (2008).

[30] G. De Chiara and G. M. Palma, Phys. Rev. Lett. 91, 090404 (2003).

[31] S. L. Zhu and P. Zanardi, Phys. Rev. A 72, 020301(R) (2005).

[32] S. Berger, M. Pechal, A. A. Abdumalikov, C. Eichler, L. Steffen, A. Fedorov, A. Wallraff, and S. Filipp, Phys. Rev. A 87, 060303(R) (2013).

[33] S. Shevchenko, S. Ashhab, and F. Nori, Phys. Rep. 492, 1 (2010).

[34] B. Damski and W. H. Zurek, Phys. Rev. A 73, 063405 (2006).

[35] W. D. Oliver, Y. Yu, J. C. Lee, K. K. Berggren, L. S. Levitov, and T. P. Orlando, Science 310, 1653 (2005).

[36] M. Sillanpää, T. Lehtinen, A. Paila, Y. Makhlin, and P. Hakonen, Phys. Rev. Lett. 96, 187002 (2006).

[37] J. R. Petta, H. Lu, and A. C. Gossard, Science 327, 669 (2010).

[38] G. Sun, X. Wen, B. Mao, J. Chen, Y. Yu, P. Wu, and S. Han, Nat. Commun. 1, 51 (2010).

[39] S. Gasparinetti, P. Solinas, and J. P. Pekola, Phys. Rev. Lett. 107, 207002 (2011).

[40] J. M. Martinis, S. Nam, J. Aumentado, and C. Urbina, Phys. Rev. Lett. 89, 117901 (2002).

[41] See Supplemental Material at http://link.aps.org/ supplemental/10.1103/PhysRevLett.112.027001 for the truncated Hamiltonian of the flux-biased phase qubit, the decoherence time of the system, and the time evolution of population $P_{1}$.

[42] The spin-echo pulse divides the process between two LZTs into two symmetric segments. The dynamical phase accumulated in each segment, which is proportional to the shaded area between points $M$ and $F$ in Fig. 1(a), are equal in magnitude but have opposite signs. Therefore, the dynamical phases between two LZs vanish.

[43] Y. Kayanuma, Phys. Rev. A 55, R2495 (1997).

[44] G. Sun, X. Wen, B. Mao, Y. Yu, J. Chen, W. Xu, L. Kang, P. Wu, and S. Han, Phys. Rev. B 83, 180507(R) (2011).

[45] R. W. Simmonds, K. Lang, D. Hite, S. Nam, D. Pappas, and J. Martinis, Phys. Rev. Lett. 93, 077003 (2004).

[46] S. Gustavsson, J. Bylander, and W. D. Oliver, Phys. Rev. Lett. 110, 016603 (2013). 